\title{
An Analysis of the Stress-Relaxation Curve obtained from a Sample of Raw Meat Sample
}

\author{
Naomichi Iso,*1 Haruo Mizuno, ${ }^{* 1}$ Takahide SArto*1, \\ Fumiaki OHZEKI, ${ }^{* 1}$ and Ching Yang LIN*1
}

(Accepted September 29, 1982)

\begin{abstract}
Large amounts of scattered results of the stress-relaxation experiments are obtained on the raw meat sample because of the heterogeneous structures and composition of the raw meat. From the computer simulation, it was shown that the probable mean values of the rheological parameters from such a stress-relaxation experiment could be obtained by making a synthetic stressrelaxation curve and analyzing the curve. The conclusion was confirmed by the experiment using composite materials.
\end{abstract}

Stress-relaxation experiments are used extensively in studies conducted on the rheological properties of viscoelastic materials, and often yield much useful information. In food science, many reports on the viscoelastic properties of foodstuffs (e.g., kamaboko, ${ }^{1)}$ dried cuttle-fish, $\left.{ }^{2}\right)$ marshmallow, ${ }^{8}$ ice-cream, ${ }^{4)}$ fruits, ${ }^{5)}$ dough, ${ }^{8}$ ) milk curd, ${ }^{7)}$ and minced meat ${ }^{8)}$ ) are published on the basis of the data from the stress-relaxation experiments.

However, when we treat raw meats as samples, the stress-relaxation curves obtained using the same sample are different from each other because of the heterogeneous structure and composition of the raw meat. Namely, the large amounts of scatter of the results of the stress-relaxation experiments on the raw meat sample come from the fact that the composition and structure of the sample varies with position. It is just like that the plunger pushes the other substance at each stress-relaxation measurement. As an example, Fig. 1 shows the stress-relaxation curves which were obtained with a raw fish meat. This gives us a serious problem to be solved before attempting to discuss the rheological properties of the raw meat sample on the basis of the stress-relaxation data. Thus, we must know how to treat the complicated data obtained from the stress-relaxation experiments on such a sample, and it is necessary to consider the experimental results statistically. In other words, we must investigate the most probable mean values of rheological parameters of such a sample.

In this study, the computer simulation method

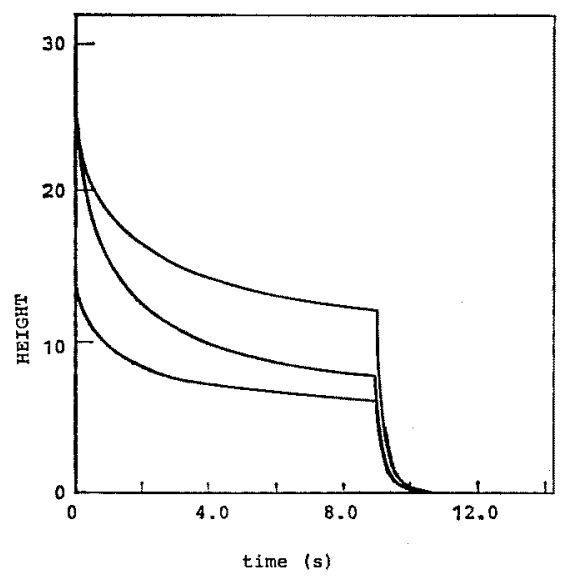

Fig. 1. The stress-relaxation curves obtained on the same carp muscle. The value of ordinate is proportional to stress.

was used to solve the problem. The applicability of the result was later confirmed by the experiment using composite materials.

\section{Experimental}

Analysis of Elastic Modulus and Viscosity

The method of analysis of the stress-relaxation curve is the progressive approximate method, ${ }^{9}$ ) which was used by SATO and NAKAYAMA ${ }^{73}$ to obtain the rheological parameters of minced meats.

The approximate equation of the stress-relaxation curve can be expressed as follows:

$$
p(t)=e_{0}\left(\sum_{i=1}^{n} E_{i} e^{-t / r_{i}}\right)
$$

*1 Department of Food Science and Technology, Tokyo University of Fisheries, Konan, Minato, Tokyo 108, Japan (磯 直道・水野治夫・齐藤隆英・大関文照・林穈陽：東京水産大学). 
where $p(t)$ is stress, $e_{0}$ the constant strain, $t$ the time, $E_{i}$ the elastic modulus of $i$-th element $\left(E_{0}=\right.$ $E_{1}+E_{2}+\cdots+E_{n}$, the instantaneous modulus), and $\tau_{i}$ the stress-relaxation time of $i$-th element $\left(\tau_{i}=\eta_{i} / E_{i}\right.$, and $\eta_{i}$ is the viscosity of $i$-th element). When $\ln p(t)$ is plotted against $t$, a straight line is obtained at large values of $\tau$. The first term of relaxation time, $\tau_{2}$, which means the largest relaxation time, and the 1-st term of elastic modulus, $E_{1}$, can be calculated from the slope of the straight line. Then the value of $\eta_{1}$ is calculated from the values of $E_{1}$ and $\tau_{1}$. Once the values of $E_{1}$ and $\tau_{1}$ are determined, $\ln \left(p(t)-e_{0} E_{1} e^{-t / \tau_{1}}\right)$ is plotted against $t$. Again a straight line is obtained at large values of $\tau$. Then the 2-nd term of relaxation time, $\tau_{2}$, and the 2-nd term of elastic molulus $E_{2}$ can be calculated from the slope of the straight line. Thus the viscosity $\eta_{2}$ can be calculated as before. The same procedures are followed till the calculated curve using the parameters obtained coincides to the experimental stress-relaxation curve. In general, two or three trials give a satisfactory result as shown in Fig. 2.

\section{Computer Simulation}

The computer used in this study was a FACOM$140 \mathrm{M}$ computer.

In this simulation, the n-value in eq. (1) is designed to be 3 . Other values of the parameters of eq. (1) are designed as follows:

$$
\begin{aligned}
& e_{0}=1 \\
& E_{0}=3.0 \times 10^{5} \mathrm{dyne} / \mathrm{cm}^{2} \\
& E_{1}=1.0 \times 10^{5} \mathrm{dyne} / \mathrm{cm}^{2}
\end{aligned}
$$

$$
\begin{aligned}
& E_{2}=1.0 \times 10^{5} \mathrm{dyne} / \mathrm{cm}^{2} \\
& E_{3}=1.0 \times 10^{5} \mathrm{dyne} / \mathrm{cm}^{2} \\
& \eta_{1}=5.0 \times 10^{6} \text { poise } \\
& \eta_{2}=5.0 \times 10^{5} \text { poise } \\
& \eta_{3}=5.0 \times 10^{4} \text { poise }
\end{aligned}
$$

These values were selected in accordance with the similar order of the viscoelastic materials. Each of the values was modified by giving the deviation $(0- \pm 30 \%)$ which was chosen at random by the computer. Using these parameters with some deviation, a stress-relaxation curve was drawn by computating the value of $p(t)$ at any value of $t$ with intervals of $0.5 \mathrm{~s}$ according to eq. (1). A total of twenty simulated stress-relaxation curves were constructed by using the twenty sets of modified parameters in this work. Although they have the same designed elastic modulus and viscosities, as shown in Fig. 3, these curves are different from each others because of the given deviation. The twenty simulated curves were analyzed by the progressive approximate method described above. Then the values were averaged as follows:

procedure (1): $\bar{x}_{1}=\sum_{i=1}^{n} x_{i} / n$ ( $x$ is the parameters, i.e. elastic modulus, viscosity, or stress-relaxation time. $n$ is the number of curve, 20.)

$$
\begin{array}{ll}
\text { procedure (2): } & \bar{x}_{2}=n / \overline{x_{1} x_{2} x_{3} \cdots x_{n}} \\
\text { procedure (3): } & \bar{x}_{\mathrm{a}}=1 /\left(\sum_{i=1}^{n}\left(1 / x_{i}\right) / n\right)
\end{array}
$$

procedure (4): $\bar{x}_{4}$ (The mean values were obtained from the synthetic curve. Namely, a synthetic stress-relaxation curve was constructc from the twenty simulated curves by averaging gra-

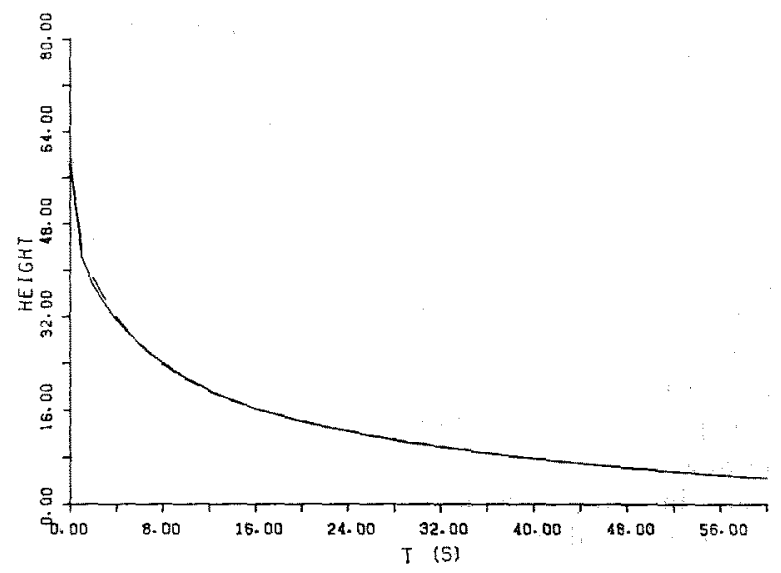

Fig. 2. The analyzing results with the progressive approximate method. The solid line is drawn using the designed rheological parameters, and the dotted line is drawn using the rheological parameters obtained by the two trials $(n=2)$ of the progressive approximate method. The value of ordinate is proportional to stress, 


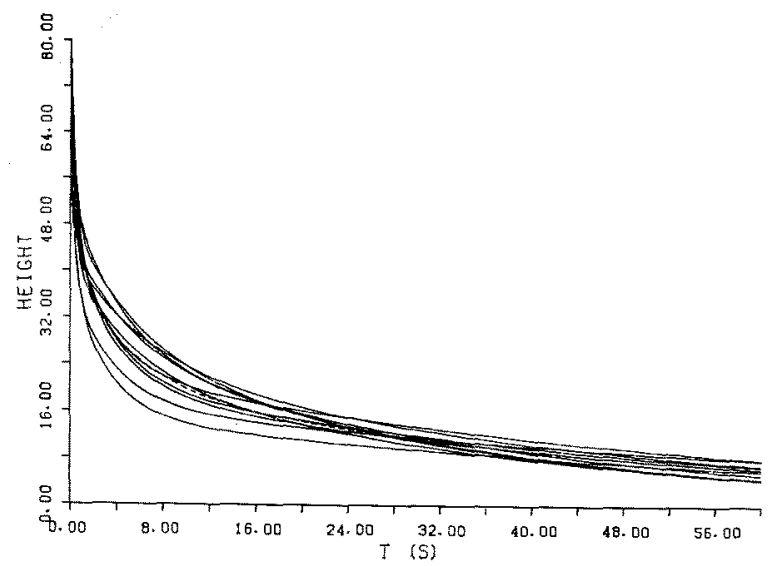

Fig. 3. The simulated stress-relaxation curves. The dotted line is drawn using the designed rheological parameters. The other solid lines are drawn by using the modified parameters. The value of ordinate is proportional to stress.

Table 1. The rheological parameters of the raw materials

\begin{tabular}{lrrrrrrr}
\hline & $E_{0}$ & $E_{1}$ & $E_{2}$ & $\eta_{1}$ & $\eta_{2}$ & $\tau_{1}$ & $\tau_{2}$ \\
\hline yokan & 13.47 & 5.00 & 8.47 & 65.1 & 3.70 & 12.9 & 0.43 \\
kamaboko & 9.81 & 8.08 & 1.73 & 44.3 & 1.32 & 54.8 & 0.77 \\
konnyaku & 2.97 & 2.47 & 0.51 & 25.8 & 0.29 & 100.6 & 0.56 \\
\hline
\end{tabular}

$E_{0}, E_{1}, E_{2} \times 10^{-5}\left(\right.$ dyne $\left./ \mathrm{cm}^{2}\right) ; \eta_{1}, \eta_{2} \times 10^{-6}$ (poise); $\tau_{1}, \tau_{2}(\mathrm{~s})$

phically and analyzed by the progressive method.)

\section{Confirmation with the Composite Materials}

As described before, the large amounts of scatter of the results of the stress-relaxation experiments on the raw meat sample come from the fact that the composition and structure of the sample are different by position. In other words, it can be said that the plunger pushes the other substance at each trial time in the experiment. The raw meat is considered to be one kind of composite material from a view point of the heterogeneity about the structure and the composition. So, we made the composite materials with the three raw materials of which the rheological parameters were decided experimentally. The values of rheological parameters obtained experimentally on the composite materials were compared with the mean values estimated from those values of the raw materials.

The composite material was made of yokan, kamaboko, and konnyaku. The stress-relaxation time are ca. 10, 50, and $100 \mathrm{~s}$, respectively. The rheological parameters of these raw materials were experimentally determined at first. The results are summarized in Table 1 . The broad thin pieces of these materials of $3 \mathrm{~mm}$ thickness were piled on top of one another. Six kinds of the composite materials were made from the three raw materials. For each kind of composite material, the stress-relaxation experiments were tried five times. Then a total of thirty stressrelaxation curves were experimentally obtained for the composite materials. The stress-relaxation experiments were carried out by using a TENSIPRESSOR Model TTP-50 BX. The type of the plunger was a cylinder of which diameter was $2.5 \mathrm{~cm}$. The sample was pushed stationally. Finally, the results obtained on the composite material were compared with the mean values estimated from the rheological parameters of the raw materials.

\section{Results and Discussion}

Table 2 shows the results of the computer simulation. The arithmetic means, $\bar{x}_{1}$, which was given by procedure (1), have the most large deviation. On the whole, procedure (4) is better than the other three procedures. Namely, the $\tilde{x}_{4}$ values are obtained from a synthetic stress-relaxation curve and have the samller deviation from the designed values except of the value of $\tau_{1}$ than those obtained by the other procedures. On the other 
Table 2. The results of several averaging procedures

\begin{tabular}{cccccccccccc}
\hline & $E_{0}$ & $E_{1}$ & $E_{2}$ & $E_{3}$ & $\eta_{1}$ & $\eta_{2}$ & $\eta_{3}$ & $\tau_{1}$ & $\tau_{2}$ & $\tau_{8}$ \\
\hline $\bar{x}_{1}$ & 9.7 & 3.5 & 3.0 & 3.2 & 11 & 14 & 8.5 & 47.7 & 4.2 & 0.3 \\
$\bar{x}_{2}$ & 5.4 & 2.3 & 1.6 & 1.5 & 6.7 & 6.7 & 4.1 & 45.8 & 4.1 & 0.3 \\
$\bar{x}_{3}$ & 3.9 & 1.6 & 1.2 & 1.1 & 5.2 & 4.8 & 2.9 & 44.1 & 4.0 & 0.3 \\
$\bar{x}_{4}$ & 2.9 & 0.9 & 1.0 & 1.0 & 4.1 & 4.4 & 3.0 & 44.8 & 4.4 & 0.4 \\
$x$ & 3.0 & 1.0 & 1.0 & 1.0 & 5.0 & 5.0 & 5.0 & 50.0 & 5.0 & 0.5 \\
\hline
\end{tabular}

$x$ : the designed value

$E_{0}, E_{1}, E_{2}, E_{8} \times 10^{-5}\left(\mathrm{dyne} / \mathrm{cm}^{2}\right) ; \eta_{1} \times 10^{-6}, \eta_{2} \times 10^{-5}, \eta_{8} \times 10^{-4}$ (poise); $\tau_{1}, \tau_{2}, \tau_{3}$ (s)

Table 3. The rheological parameters of the composite material and the mean values of the raw materials

\begin{tabular}{|c|c|c|c|c|c|c|c|}
\hline & $E_{0}$ & $E_{\text {x }}$ & $E_{2}$ & $\eta_{1}$ & $\eta_{2}$ & $\tau_{1}$ & $\tau_{2}$ \\
\hline \multicolumn{8}{|l|}{ composite } \\
\hline material & 7.7 & 4.8 & 2.9 & 12.8 & 1.7 & 26.6 & 0.6 \\
\hline $\bar{x}_{1}$ & 9.1 & 5.2 & 4.0 & 46.3 & 1.9 & 52.8 & 0.6 \\
\hline $\bar{x}_{2}$ & 6.8 & 4.7 & 2.2 & 42.7 & 1.2 & 37.8 & 0.6 \\
\hline$\underline{x}_{9}$ & 5.4 & 4.2 & 1.2 & 39.2 & 0.7 & 25.9 & 0.5 \\
\hline $\bar{x}_{4}$ & 8.2 & 5.2 & 3.0 & 45.2 & 1.6 & 45.2 & 0.6 \\
\hline
\end{tabular}

hand, the values of $\bar{x}_{1}$ (arithmetic mean), $\bar{x}_{2}$ (geometric mean), and $\bar{x}_{8}$ (reciprocal of the reciprocal mean) have large deviations from the designed values.

Table 3 shows the rheological parameters of the composite material and the mean values $\left(\bar{x}_{1}\right.$, $\bar{x}_{2}, \bar{x}_{3}$, and $\bar{x}_{4}$ ) of the rheological parameters of the three raw materials. The $\eta_{1}$ values are quite different from those on the composite material in all cases. However, as a whole, procedure (4) gives better results. It confirms the result of the computer simulation.

On the basis of these results from the computer simulation and the evidence from the experiments using the composite materials, we conclude as follows: in the determination of the rheological parameters from some different stress-relaxation curves which are obtained on the same sample, a synthetic stress-relaxation curve should be constructed at first and then analyzed in order to obtain the rheological parameters. Another approach, i.e. the way that the individual stressrelaxation curves are analyzed at first and then the rheological parameters obtained are averaged may lead to an erroneous result.

\section{References}

1) A. KISHIMoto and E. MAEKAWA: Bull. Japan. Soc. Sci. Fish., 28, 803-808 (1962).

2) A. Kisermoto and H. Fustra: Bull. Japan. Soc. Sci. Fish., 22, 293-269 (1956).

3) W. N. Duck: Confect. Prod., 27, 153-155 (1961).

5) D. SHAW: in "Rheology of Emulsion", (ed. by P. Sherman), Pergamon Press, London, 1963, pp. $125-131$.

5) N. N. MOHSENIN and C. T. MORRow: in "Rheology and Texture of Foodstuffs", S. C. I. Monograph, No. 27, London, 1968, pp. 50-73.

6) J. GlüCKrICH and L. SheleF: Cereal. Chem., 39, 242-246 (1962).

7) F. Shama and P. Sherman: in "Rheology and Texture of Foodstuffs", S.C.I. Monograph, No. 27, London, 1968, pp. 77-90.

8) Y. Sato and T. Nakayama: J. Text. Stud., 1, 309-326 (1970).

9) A. V. Tobolsky and K. Murakami: $J$. Polymer Sci., 40, 443-446 (1959). 